\title{
Defective imitation of gestures in patients with damage in the left or right hemispheres
}

\author{
Georg Goldenberg
}

\begin{abstract}
Objectives-Defective imitation of meaningless gestures has repeatedly been demonstrated in patients with apraxia and has been interpreted as being due to a deficit of motor execution. There is, however, controversy as to whether some impairment of imitation also occurs in patients with right brain damage. The aim was to compare defective imitation in patients with left and right brain damage and to explore whether there are qualitative differences between them.
\end{abstract}

Methods-Imitation was examined in 80 patients with left brain damage (LBD) and aphasia, 40 patients with right brain damage (RBD), and 60 controls for three types of gestures: hand positions, finger configurations, and combined gestures which required a defined hand position as well as a defined configuration of the fingers.

Results-Regardless of whether imitation of hand positions and finger configurations were tested each on their own or together, they showed differential susceptibility to RBD and LBD. Whereas imitation of finger configurations was about equally impaired in RBD and LBD, defective imitation of hand positions occurred almost exclusively in patients with LBD, and whereas controls as well as patients with RBD committed less errors with hand positions than with finger configurations, the reverse was the case in patients with LBD.

Conclusions-The pattern of results goes against a deficit of motor execution as being the cause of defective imitation in patients with LBD, as it is difficult to see why such a deficit should affect proximal movements necessary for reaching hand positions more than differential finger movements. An alternative explanation would be that in patients with LBD errors are due to defective mediation by knowledge about the human body whereas in patients with RBD they stem from faulty visuospatial analysis of the demonstrated gesture.

\section{$(\Im$ Neurol Neurosurg Psychiatry 1996;61:176-180)}

Keywords: apraxia; motor control; aphasia; visuospatial abilities

Defective imitation of gestures has traditionally been recognised as a symptom of ideomo- tor apraxia. ${ }^{1-9}$ Ideomotor apraxia is a sequel of lesions to the left hemisphere and is nearly always accompanied by aphasia, but imitation of gestures is essentially a non-verbal task, the results of which are unlikely to be contaminated by aphasia. As defective imitation has been demonstrated even for meaningless and novel gestures, neither can it be referred to a general "asymbolia" which may disturb the comprehension and expression of symbolic gestures. ${ }^{10}$ The independence from the influence of language disorders and asymbolia makes imitation of gestures a test which is easy to administer even in severely aphasic patients and which promises insights into dominance of the left hemisphere for motor control beyond dominance for language and symbolic expression.

The empirical basis for recognising defective imitation of gestures as a clue to left hemispheric dominance is weakened by the finding of impaired imitation in patients with damage restricted to the right hemisphere. Probing imitation of a large sample of gestures requiring the reproduction of both the position of the hand and the configuration of the fingers, De Renzi and coworkers repeatedly found that patients with right brain damage (RBD) commit more errors than controls albeit their impairment is less severe than that of patients with left hemispheric damage (LBD). ${ }^{46}$ Kimura and Archibald found virtually no difference between patients with LBD and those with RBD when imitation was tested for configurations of the fingers of the hand, whereas imitation was significantly worse in patients with LBD than in patients with RBD when tested for movement sequences which defined the positions of the hand relative to the body as well as the configurations of the fingers. ${ }^{3}$ Lehmkuhl et al found defective imitation in patients with LBD but not in patients with RBD. 9 The meaningless gestures for which they tested imitation specified only hand positions and did not require particular configurations of the fingers.

From these studies it might be deduced that the susceptibility of imitation to RBD and LBD depends on whether the reproduction of hand positions or of configurations of the fingers is required. However, none of the studies explicitly considered this distinction, and in each of them differences between hand positions and finger configurations were obtunded by other factors likely to influence the success of imitation-for example, differences between symbolic and meaningless gestures or between static gestures and movement sequences. 
The purpose of the present study was to systematically compare imitation of hand positions and finger configurations between patients with LBD, patients with $\mathrm{RBD}$, and controls and to explore whether differences between hand positions and finger configurations provide clues to the mechanisms of defective imitation in LBD and RBD.

\section{Materials and methods}

METHOD OF TESTING

Imitation was tested for three different kinds of meaningless gestures (fig 1): imitation of hand postures required the patients to copy different positions of the hand relative to the head while the configuration of the fingers remained invariant. For imitation of finger postures patients were asked to replicate different configurations of the fingers. The position of the whole hand relative to the body was not considered for scoring. Combined gestures required both a defined position of the hand relative to the body and defined configuration of the fingers.

The procedure of testing and scoring was the same for all three types of postures. The examiner sat opposite the patient and demonstrated the gesture "like a mirror". If the patient used the left hand, the examiner demonstrated with the right hand and vice versa. The patients were allowed to start imitation as soon as the demonstration was terminated. For a correct imitation on first trial two points were credited. Otherwise, the demonstration was repeated and one point was given for a successful second trial. Scoring consid- ered only the final position of the hand or fingers and did not take into account hesitation, searching movements, or self corrections during the course of the movement. For combined gestures, credit was given only when both the position of the hand and the configuration of the fingers were correct, but in addition to this global scoring the position of the hand and the configuration of the fingers were scored separately. There were 10 items for each type of gesture resulting in a maximum score of 20.

All patients imitated with the hand ipsilateral to lesion, and half of the controls used the right hand and half the left.

\section{SUBJECTS}

Eighty patients with LBD, 40 patients with RBD, and 60 controls were examined. All patients with brain damage had had a first, unilateral stroke within the middle cerebral artery territory. Controls were recruited from neurological inpatients with diseases of the spine or of the peripheral nerves and without any sign or history of cerebral damage. Patients with LBD were excluded from the study if they had no aphasia at all and patients with RBD if neuropsychological assessment including the WAIS subtest block design and a copy of the Rey figure did not disclose any signs of visuospatial dysfunction. (Two nonaphasic patients with LBD in whom imitation of gestures was impaired will be reported in a separate paper.) Aphasia was classified as global in 26 patients, Broca's in 11 , Wernicke's in 16, amnesic in 16, and as transcortical, conduction, or non-classifiable
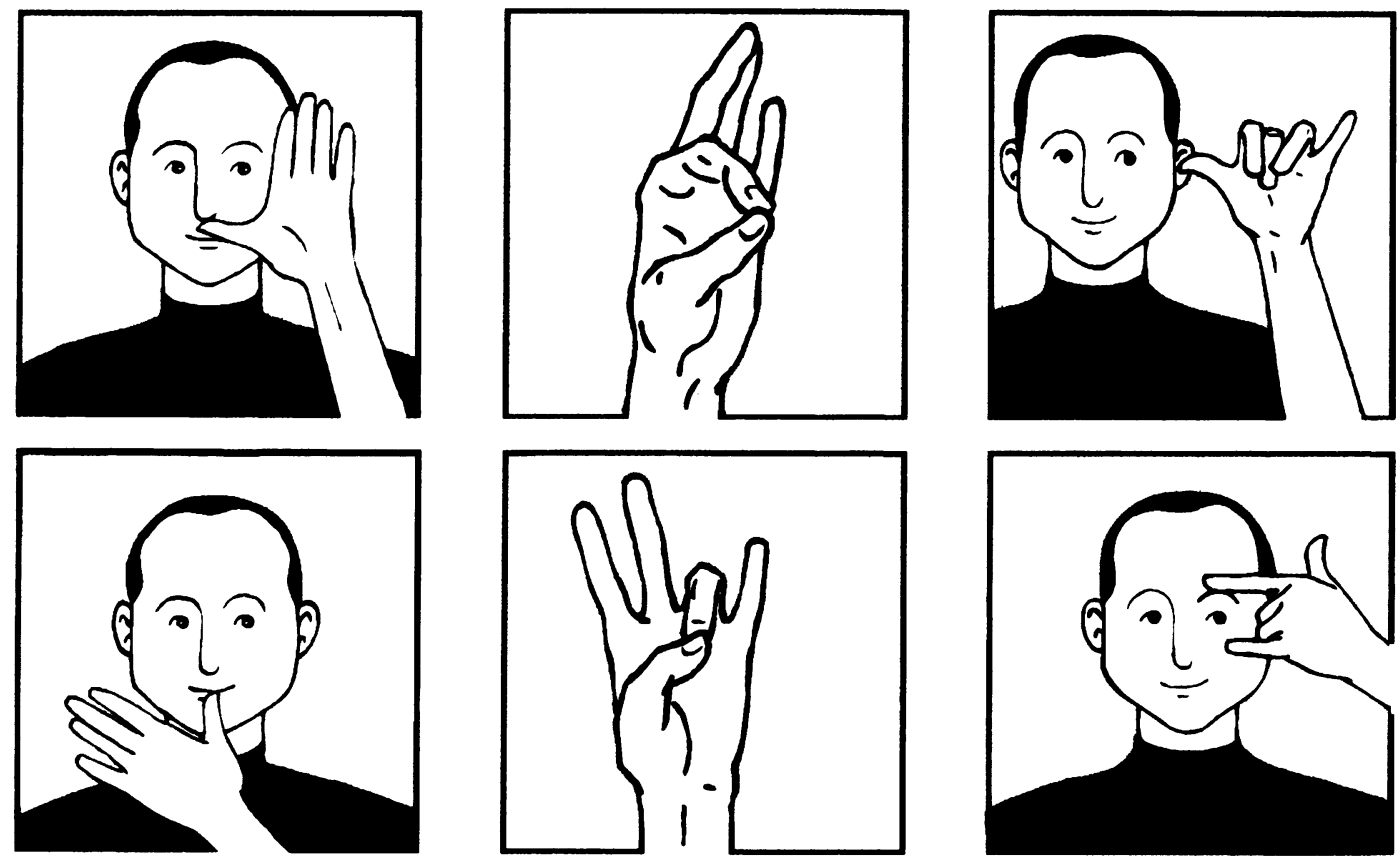

Figure 1 Examples of hand positions (left column), finger configurations (middle column), and combined gestures (right column). A complete description of the hand postures has been published. ${ }^{14}$ The dissociation between imitation of finger configuration and of hand positions was particularly striking in combined gestures. For example, in the gesture shown in the upper row a typical error of patients with $L B D$ would be to touch the ear with the little finger instead of the thumb while correctly bending the middle three fingers. By contrast, a typical error of patients with RBD would be to hold the first three fingers extended and bend the forth and fifth finger while correctly touching the ear with the thumb. In the gesture shown in the lower row a typical LBD error would be to hold the correctly shap l hand before the cheek rather than before the eyes, and a typical RBD error to extend index and middle finger before the eye. In patients with LBD errors often concerned both hand positions and finger configurations. 
Table 1 Demographic, clinical, and radiological data of patients and controls

\begin{tabular}{llll}
\hline & $L B D$ & $R B D$ & Controls \\
\hline Female/male & $28 / 52$ & $18 / 22$ & $23 / 37$ \\
Age & $57 \cdot 5(14 \cdot 0)$ & $58 \cdot 4(13 \cdot 2)$ & $54 \cdot 9(14 \cdot 0)$ \\
Time since CVA (months) & $4 \cdot 1(9 \cdot 6)$ & $3 \cdot 8(7 \cdot 8)$ & - \\
Ischaemia/haemorrhage & $59 / 21$ & $25 / 15$ & - \\
Plegic/not plegic & $52 / 28$ & $26 / 14$ & - \\
Percentage of patients with lesions in: & 29 & 30 & - \\
$\quad$ Frontal lobe & 30 & 20 & - \\
Insula & 25 & 20 & - \\
Parietal lobe & 30 & 32 & - \\
Temporal lobe & 18 & 13 & - \\
Basal ganglia & 8 & 10 & - \\
Thalamus & 50 & 40 & - \\
\hline White matter & & & \\
\hline
\end{tabular}

Values in parentheses are SD. Location of lesions is based on CT, which was available for all but two patients. The sum of the percentages is more than 100 as most patients had lesions affecting several regions.
(40\%) patients with RBD scored lower than any control, and the distribution of defective scores was similar in both groups of brain damaged patients.

Imitation of combined postures proved to be the most difficult task for controls. There was no ceiling effect and the poorest performance equalled only half the maximum score. The two lowest control scores were more than 3 SD below the mean. They were considered as outliers and the cut off was set to 13 . Fifty $(63 \%)$ of the aphasic patients and $13(33 \%)$ of the patients with RBD were classified as defective. Inclusion of the two outliers would have lowered the number of patients classified as defective to $34(42.5 \%)$ and five $(12.5 \%)$ respectively. Extremely poor performance (0 and 1 point) was found only in aphasic patients.

Because of the ceiling effects described nonparametric tests were preferred for the statistical comparisons.

The left sided panel of figure 2 shows the mean scores on imitation of hand positions and finger configurations for the three groups. Controls committed significantly less errors with hand positions than with finger configurations (Wilcoxon test: $z=-3.0 ; \mathrm{p}<0.01$ ). The difference in favour of hand positions was even more marked in patients with $\operatorname{RBD}(z=$ $-5.2 ; p<0.00005)$. By contrast, patients with LBD made more errors with hand positions than with finger configurations $(z=$ $-1.97 ; \mathrm{p}<0.05)$.

The total sample of patients with brain damage included those who were not at all impaired in imitating gestures. In these patients the relation between hand and finger postures was likely to be the same as in controls. Their inclusion may have attenuated the differential sensitivity of hand and finger postures to RBD and LBD. Therefore, the analysis of the brain damaged patients' scores was repeated after exclusion of patients who had copied hand positions and finger configurations within the normal range. There remained 15 patients with $\mathrm{RBD}$ and 49 with LBD. The right panel of figure 2 shows their Twenty six (33\%) aphasic patients and 16

Table 2 Distribution of scores for imitation of hand positions, finger configurations, and combined gestures

\begin{tabular}{|c|c|c|c|c|c|c|c|c|c|}
\hline \multirow[b]{2}{*}{ Score } & \multicolumn{3}{|l|}{ Hand } & \multicolumn{3}{|l|}{ Finger } & \multicolumn{3}{|c|}{ Combined } \\
\hline & $L B D$ & $R B D$ & Control & $L B D$ & $R B D$ & Control & $L B D$ & $R B D$ & Control \\
\hline 20 & 18 & 23 & 45 & 9 & 3 & 33 & 1 & - & 8 \\
\hline 19 & 9 & 7 & 11 & 13 & 3 & 13 & 3 & 1 & 9 \\
\hline 18 & 8 & 3 & 4 & 13 & 7 & 7 & 3 & 1 & 12 \\
\hline 17 & 7 & 5 & - & 5 & 2 & 4 & 6 & 6 & 14 \\
\hline 16 & 4 & 1 & - & 8 & 8 & 2 & 7 & 4 & 8 \\
\hline 15 & 1 & 1 & - & 5 & 5 & 1 & 4 & 6 & 5 \\
\hline 14 & 3 & - & - & 2 & 2 & - & 2 & 5 & 1 \\
\hline 13 & 1 & - & - & 7 & 3 & - & 4 & 4 & 1 \\
\hline 12 & 3 & - & - & 2 & 4 & - & 4 & 4 & - \\
\hline 11 & 5 & - & - & 6 & 4 & - & 8 & 3 & 1 \\
\hline 10 & 3 & - & 一 & 1 & 1 & - & 4 & 1 & 1 \\
\hline 9 & 3 & - & - & 2 & 1 & - & 4 & 2 & - \\
\hline 8 & 5 & - & - & 1 & - & - & 8 & - & - \\
\hline 7 & 2 & - & - & 1 & - & - & 2 & - & - \\
\hline 6 & - & - & - & 1 & 1 & - & 6 & 1 & - \\
\hline 5 & 2 & - & - & - & - & - & - & 1 & - \\
\hline 4 & 1 & - & - & 3 & - & - & 1 & - & - \\
\hline 3 & 1 & - & - & - & - & - & 1 & 1 & - \\
\hline 2 & 3 & - & - & - & - & - & - & - & 一 \\
\hline 1 & - & - & - & - & - & - & 5 & - & - \\
\hline 0 & 1 & - & - & - & - & - & 7 & - & - \\
\hline
\end{tabular}

The table shows the actual number of patients achieving a score. There were 80 patients with LBD, 40 with RBD, and 60 controls. 
Figure 2 Left panel: mean scores of the three groups on imitation of hand positions and finger configurations. Right panel: mean scores of patients with $L B D$ or patients with $L B D$ or patients whose scores were normal for both types of gestures.

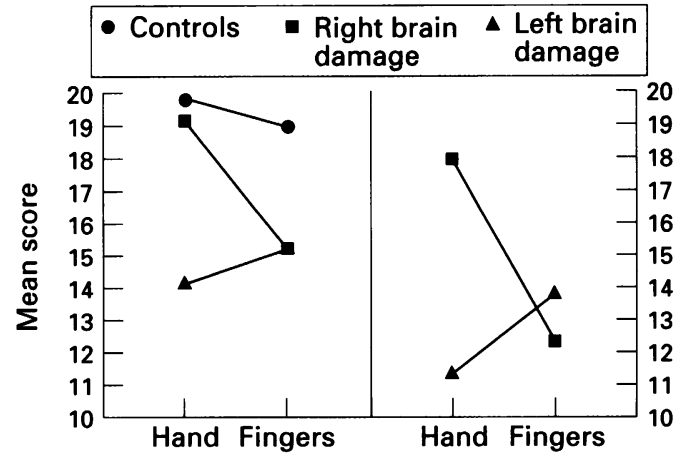

Figure 3 Left panel: mean scores of the three groups on hand positions and finger configurations within the combined gestures. Right panel: mean scores of patients with $L B D$ or $R B D$ after exclusion of patients whose combined score was above the cut off for controls.

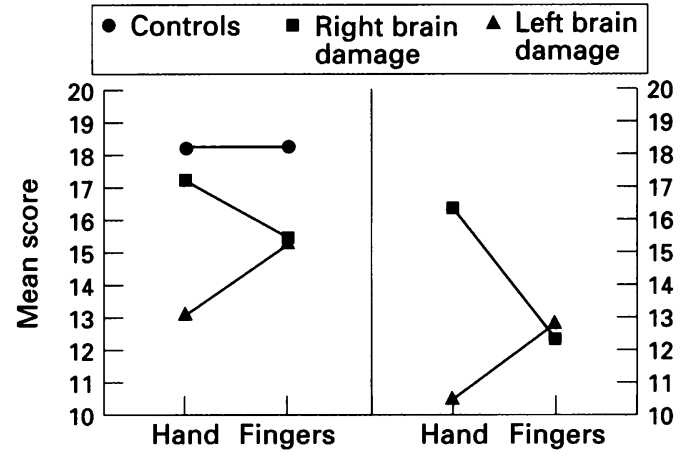

mean scores. In both groups the exclusion of patients without any impairment amplified the differences between hand positions and finger configurations. In patients with $\mathrm{RBD}$ the mean difference in favour of hand positions increased from 4.0 to 5.6 , whereas in aphasic patients the reverse difference increased from $1 \cdot 0$ to $2 \cdot 3$

Figure 3 shows the separate analyses of the position of the hand and the configuration of the fingers in combined gestures. There was hardly any difference between hand positions and finger configurations in controls $(z=$ $-0 \cdot 4 ; p>0 \cdot 5)$. Patients with RBD committed more errors with finger configurations than with hand positions $(z=-3.2 ; \mathrm{p}<0.01)$ whereas the opposite was the case in patients with LBD $(z=-2 \cdot 8 ; \mathrm{p}<0.01)$. The right hand panel of fig 3 shows the results of only those brain damaged patients whose global score for combined gestures was below the cut off point. For patients with RBD the mean difference in favour of hand positions increased from $2 \cdot 0$ to $4 \cdot 0$, whereas for aphasic patients the reverse difference increased from 1.8 to $2 \cdot 3$. If the analysis was confined to only those brain damaged patients whose combined scores fell below those of the control classified as outliers, the differences increased to 5.6 for patients with RBD and to 2.5 for the aphasic patients.

From the type of errors it seemed that visual feedback control of motor execution did not play a major role in superior performance of patients with LBD with finger configurations. This was particularly evident in combined gestures. Even when the hand position was wrong, the hand was most often raised to the level of the head and held beside the head or touching the face. Consequently, the finger configuration was hidden from direct view as much as it would have been with the correct hand position (see fig 1). None the less, finger configuration was often correct whereas hand position was not.

\section{Discussion}

Regardless of whether imitation of hand positions and finger configurations were tested each on their own or together, they showed differential susceptibility to RBD and LBD. Whereas imitation of finger configurations was about equally impaired in RBD and LBD, defective imitation of hand positions occurred almost exclusively in patients with LBD, and whereas controls as well as patients with RBD committed less errors with hand positions than with finger configurations, the reverse was the case in patients with LBD. The opposite differences between hand positions and finger configurations in patients with LBD and those with RBD indicate that different mechanisms cause defective imitation in both groups.

Analyses of the task demands of imitation have been embedded in theories of ideomotor apraxia. They follow Liepmann's original proposal that faulty imitation by apraxic patients is due to an inability to implement the correctly conceived gesture into an appropriate motor act-that is, a deficit of motor execution. ${ }^{161112}$ The very term "ideomotor apraxia" implicates this interpretation: it denotes an inability to translate a correct idea of the intended gesture into a motor act and hence indicates defective motor execution as the source of apractic errors. ${ }^{1}$ The results of the present study are not easily accommodated by this conjecture. It is difficult to see why a deficit of motor execution should affect the movements of the whole upper limb necessary for reaching hand positions more than the differential movements of single fingers necessary to assume finger configurations.

A more satisfactory account for the particular difficulties of patients with LBD with the copying of hand position could be based on the hypothesis that knowledge about the human body mediates the transition from visual perception to motor execution. ${ }^{21314}$ This knowledge is not restricted to awareness of the current extension and configuration of a person's own body but applies to the invariant structure of any human body. It contains a generally valid structural description of the human body which provides a classification of significant body parts and specifies the boundaries that define them..$^{15}$ Its application reduces the multiple details of the demonstrated gesture to simple relations between a limited number of significant body parts which can easily be maintained in working memory from the end of the demonstration until the accomplishment of motor execution ${ }^{\star}$ and

*The instruction to start imitation only immediately after the demonstration required storage of the gesture in working memory. It is, however, questionable whether a persistence of the demonstration during imitation would significantly reduce the demands on working memory. The simultaneous evaluation of the demonstrated gesture and its imitation is likely to put another load on working memory, the weight of which would also depend on how efficiently the critical features of the gesture are coded. 
which may have privileged access to routine motor programmes. ${ }^{16}$ As a further advantage, translating the gesture's visual appearance into the categories of a generally valid concept of the human body produces an equivalence between demonstration and imitation which is independent of the particular angle of view under which the demonstration is perceived.

The demands for mediation by general knowledge about the human body could be different for imitation of hand positions and finger configurations. Hand positions were defined with respect to the face and head. A person's own head and face can be perceived only indirectly and not in the same way as those of another person. Consequently, the mental representation of a person's own proximal body parts is different from the mental representation of the same parts on another person's body. Common coding by the generally valid concept of the human body is necessary to mediate the equivalence between these different mental representations of the human body when imitation requires the translation of a gesture from the examiner's body to the patient's own body. By contrast, a person's own hand is often perceived in a very similar way to the hand of another person. The mental representation of a person's own finger configuration may not be much different from that of another person's finger configuration. This direct similarity between the mental representations of perceived and imitated finger configurations could partially bypass mediation by generally valid knowledge about the human body.

Another difference between hand positions and finger configurations concerns the demands on visuospatial analysis of the perceived gesture. Hand positions were determined by relations between perceptually distinct body parts such as the mouth, nose, or ear and, on the hand itself, the back and the palm or the ulnar and the radial edge. By contrast, fingers are perceptually similar. Their identity is mainly determined by their spatial position with respect to the other fingers and to the hand. ${ }^{17}$ Exact visual exploration and visuospatial analysis of the perceived finger configuration are thus a prerequisite for application of the generally valid concept of the human body.

Starting from these considerations the following interpretation of the results of the present study might be put forward: whereas in patients with LBD mediation by knowledge about the human body is defective, patients with $\mathrm{RBD}$ have difficulties with the visuospatial analysis of the demonstrated gesture. Consequently, patients with RBD have more difficulties with finger configurations than with hand position. Patients with LBD commit errors with both types of gestures but because of the possibility of bypassing the concept of the human body in the imitation of finger configurations, these are less affected than hand positions.

Studies which examined pointing to body parts lend support to the notion that both hemispheres make different contributions to the control of movements depending on whether the targets are defined in relation to proximal body parts or in relation to the fingers. In these studies patients were asked to point on themselves to body parts shown on a model of the human body or, conversely, to point on a model to the body parts touched on themselves. When this was done for the face, the head, the trunk, and the large joints of the limbs, patients with LBD committed errors whereas patients with RBD performed flawlessly. ${ }^{418}$ By contrast, when the test asked for the identification of single fingers, the error rates were the same in patients with LBD or RBD. ${ }^{19}$

The proposal that defective imitation of meaningless gestures stems from an inability to apply general knowledge about the human body in patients with $\mathrm{LBD}$, and from defective visuospatial processing in patients with $R B D$ has clinical and theoretical implications: for clinical practice, it implies that imitation of hand positions and of finger configurations are two basically different tasks, the results of which should not be confounded by compound scores. For the theory of hemispheric dominance it implies that defective imitation in patients with ideomotor apraxia points to a left hemispheric dominance for the application of a generally valid concept of the human body but does not prove a left hemispheric dominance for motor execution.

The investigation was performed when I was at the 2nd Neurological Department of the Hospital Rosenhügel, Vienna, Austria, and was supported by project 8910 MED from the Austrian Science Foundation. I thank Professor Bruno Mamoli for the possibility of examining patients under his care Joseph Sor the possibility of examining patients under his care, Joseph Spatt and Sonja Hagmann for assistance in examinations and discussions of the result

1 Liepmann H. Drei Aufsätze aus dem Apraxiegebiet. Karger: Berlin, 1908

2 Morlaas J. Contribution à l'étude de l'apraxie. Paris: Amédée Legrand, 1928.

3 Kimura D, Archibald Y. Motor functions of the left hemisphere. Brain 1974;97:337-50.

4 DeRenzi E, Motti F, Nichelli P. Imitating gestures-a quantitative approach to ideomotor apraxia. Arch Neurol 1980;37:6-10.

5 de Renzi E. Apraxia. In: Boller F, Grafman J, eds. Handbook of clinical neuropsychology. Vol 2. Oxford: Elsevier, 1990:245-63.

6 Barbieri C, de Renzi E. The executive and ideational components of apraxia. Cortex 1988;24:535-44.

7 Heilman KM, Rothi LJG. Apraxia. In: Heilman KM Valenstein E, eds. Clinical neuropsychology. Oxford: Oxfenstein E, eds. Clinical neuropsycho

8 Roy EA, Square-Storer P, Hogg S, Adams S. Analysis of task demands in apraxia. Int $\mathcal{F}$ Neurosci 1991;56:177-86.

9 Lehmkuhl G, Poeck K, Willmes K. Ideomotor apraxia and aphasia: an examination of types and manifestations of apraxic symptoms. Neuropsychologia 1983;21:199-212.

10 Finkelnburg FC. Sitzung der Niederrheinischen Gesellschaft in Bonn. Medizinische Section. Berliner Klinische Wochenschrift 1870;7:449-50;460-2.

11 Rothi LJG, Ochipa C, Heilman KM. A cognitive neuropsychological model of limb praxis. Cognitive Neuropsychology 1991;8:443-58.

12 Roy EA, Hall C. Limb apraxia: a process approach. In: Proteau L, Elliott D, eds. Vision and motor control. Amsterdam: Elsevier, 1992:261-82.

13 de Ajuriaguerra J, Tissot R. The apraxias. In: Vinken PJ, Bruyn GW, eds. Handbook of clinical neurology. Vol 4. Bruyn GW, eds. Handbook of clinical neur
Amsterdam: North Holland, 1969:48-66.

14 Goldenberg $G$. Imitating gestures and manipulating a mannikin - the representation of the human body in ideomotor nikin-the representation of the human body

15 Sirigu A, Grafman J, Bressler K, Sunderland T. Multiple representations contribute to body knowledge processrepresentations contribute to

16 Goldenberg G, Hermsdörfer J, Spatt J. Ideomotor apraxia and cerebral dominance for motor control. Cognitive Brain Research 1996;3:95-100.

17 Stengel E. Loss of spatial orientation, constructional apraxia and Gerstmann's syndrome. Fournal of Mental Science 1944;90:753-60.

18 Semenza C, Goodglass $\dot{H}$. Localization of body parts in brain injured subjects. Neuropsychologia 1985;23:161-76.

19 Gainotti G, Cianchetti C, Tiacci C. The influence of the hemispheric side of lesion on non verbal tasks of finger localization. Cortex 1972;8:364-81. 